Article

\title{
Effect of Several Nutrients and Environmental Conditions on Intracellular Melatonin Synthesis in Saccharomyces cerevisiae
}

\author{
María Ángeles Morcillo-Parra ${ }^{(D)}$, Gemma Beltran ${ }^{\circledR}$, Albert Mas ${ }^{\circledR}$ and María-Jesús Torija *(D) \\ Grup de Biotecnologia Enològica, Departament de Bioquímica i Biotecnologia, Facultat d'Enologia, \\ Universitat Rovira i Virgili, c/Marcel-lí Domingo, 1-43007 Tarragona, Spain; \\ maangeles.morcillo@urv.cat (M.Á.M.-P.); gemma.beltran@urv.cat (G.B.); albert.mas@urv.cat (A.M.) \\ * Correspondence: mjesus.torija@urv.cat
}

Received: 18 May 2020; Accepted: 3 June 2020; Published: 5 June 2020

check for updates

\begin{abstract}
Melatonin is a bioactive compound that is present in fermented beverages and has been described to be synthesized by yeast during alcoholic fermentation. The aim of this study was to assess the capacity of intracellular and extracellular melatonin production by different Saccharomyces strains from diverse food origin and to study the effects of different fermentation parameters, such as sugar and nitrogen concentration, temperature or initial population, on melatonin production using a synthetic grape must medium. Melatonin from fermentation samples was analyzed by liquid chromatography mass spectrometry. Intracellular melatonin synthesis profile did not present differences between yeast strains. However, extracellular melatonin production depended on the yeast origin. Thus, we suggest that melatonin production and secretion during the different yeast growth phases follows a species-specific pattern. Other parameters that affected the fermentation process such as sugar content and low temperature had an impact on intracellular melatonin production profile, as well as the melatonin content within the cell. This study reports the effect of several conditions on the melatonin synthesis profile, highlighting its possible role as a signal molecule.
\end{abstract}

Keywords: melatonin; alcoholic fermentation; yeast; temperature; Saccharomyces cerevisiae

\section{Introduction}

Several factors, either biotic or abiotic, can affect the yeasts present during alcoholic fermentation, and thus, modify the fermentation performance and the production of bioactive compounds [1,2]. The microbial interactions and responses, such as the synthesis of antimicrobial compounds, competition for nutrients and yeast-yeast cell contact are the main biotic factors [3,4]. On the other hand, abiotic factors are defined as environmental aspects that affect the winemaking process, such as nutrients (sugar and nitrogen), temperature and $\mathrm{pH}$, among others [5]. Sugar content in grape must is the one of the first stresses that yeasts have to deal with. This high sugar content derives in osmotic shock, and the yeast response is to modify the cell wall and the cytoskeleton as well as to activate the synthesis of glycerol to reestablish the osmotic balance [6,7]. Nitrogen is also an important and critical nutrient for yeast cells. In fact, low nitrogen levels can result in stuck or sluggish fermentation [8]. Furthermore, temperature can also alter the development of fermentation, specifically yeast growth $[9,10]$.

Melatonin is a bioactive molecule that has been recently described to have a positive role against oxidative stress [11,12] and UV stress [13] in yeasts. In addition to its protective role, melatonin is produced by yeast during the winemaking process [14-17].

Since Sprenger and collaborators [18] observed intracellular melatonin after tryptophan pulse when yeast cells were arrested in minimal medium, many studies have focused on finding this molecule 
in fermented beverages such as wine or beer [14,19-21]. Rodriguez-Naranjo and collaborators [22] highlighted the role of yeast, specifically Saccharomyces cerevisiae, in the production of melatonin during the winemaking process. However, not only does S. cerevisiae produce melatonin but also several non-Saccharomyces strains are able to synthetize melatonin during alcoholic fermentation [14-16,23-25]. Melatonin production depends on the availability of tryptophan, which is a precursor [14,16,26], but also some tryptophan derivatives ( $\mathrm{N}$-acetyl serotonin or 5-methoxytryptamine) can be used as melatonin precursors [27]. However, other fermentation conditions, such as growth medium composition (reducing sugars or nitrogen content) and yeast growth phase, can affect melatonin synthesis [25,26]. Moreover, melatonin content is positively correlated with the ethanol production rate during alcoholic fermentation, suggesting that melatonin could participate in alcoholic fermentation [28].

Melatonin has been described in the extracellular medium during the exponential phase of growth (between the first and second day) $[14,15,17,23,24,26]$ and at the end of alcoholic fermentation (at the stationary phase) [25] depending on the yeast species involved in the fermentation. Nevertheless, few studies about intracellular melatonin have been published. Some authors have provided evidence that melatonin is present intracellularly after precursor pulses $[16,18,27]$, and also, in oenological conditions, both in Saccharomyces [17] and in non-Saccharomyces yeasts [16,24], although few strains have been studied.

The aim of this study was (i) to assess the capacity of intra- and extracellular melatonin production by different strains of Saccharomyces yeasts and (ii) to study the effects of different nutrient and environmental conditions (sugar, nitrogen, temperature, inoculum and cell synchronization) on melatonin production in order to understand the conditions required for the production of melatonin by yeast.

\section{Materials and Methods}

\subsection{Yeast Strain and Inoculum Preparation}

In this study, we used three different strains of S. cerevisiae, QA23 (from wine), Instaferm (from bread baking) and Levucell SC20 (from animal nutrition), and one strain of S. pastorianus, Diamond (from beer), which were provided by Lallemand S.A. (Lallemand Inc. Montreal, QC, Canada). The synchronization experiments were performed on the derivative haploid of the commercial strains QA23 (QA23 ho-, [29]). Dry yeasts were rehydrated in water at $37{ }^{\circ} \mathrm{C}$ for $30 \mathrm{~min}$. Afterwards, the precultures were prepared in $50 \mathrm{~mL}$ of YPD broth (1\% (w/v) yeast extract, $2 \%(w / v)$ glucose and $2 \%(w / v)$ bacteriological peptone (Panreac Quimica SLU, Barcelona, Spain)) at $28^{\circ} \mathrm{C}$ with a stirring rate of $120 \mathrm{rpm}$ in an orbital shaker. Then, yeast cells were transferred into fresh minimal medium (YNB; 1X yeast nitrogen base without amino acids or ammonia (Becton, Dickinson and Company, Sparks, MD, USA), 2\% (w/v) glucose, and $350 \mathrm{mM}\left(\mathrm{NH}_{4}\right)_{2} \mathrm{SO}_{4}$ (Panreac Quimica SLU, Barcelona, Spain)) and cultured for three days at $28^{\circ} \mathrm{C}$ and $120 \mathrm{rpm}$.

\subsection{Alcoholic Fermentation Conditions}

Synthetic grape must was prepared as in Beltran et al. [30], but with a modification on the aromatic amino acid (tryptophan, tyrosine and phenylalanine) concentration. In this study, a five-fold increase in aromatic amino acids was used at the expense of the remaining amino acids to maintain the concentration of yeast assimilable nitrogen (YAN) at $300 \mathrm{mg} \mathrm{N} / \mathrm{L}$. This synthetic grape must (with $200 \mathrm{~g} / \mathrm{L}$ sugars (1:1 glucose:fructose) and $300 \mathrm{mg} \mathrm{N} / \mathrm{L}$ ) was considered the standard medium for this study. After the preparation, the synthetic grape must was stored at $4{ }^{\circ} \mathrm{C}$ until used. In higher temperature experiments $\left(12\right.$ and $\left.28^{\circ} \mathrm{C}\right)$, the must was pre-warmed to the desired temperature before inoculation.

Two different experiments were designed. The first consisted of performing fermentations in standard medium using different S. cerevisiae strains. Yeasts (QA23, Instaferm, Levucell and Diamond) were inoculated at $2 \times 10^{6} \mathrm{cell} / \mathrm{mL}$ in $450 \mathrm{~mL}$ of synthetic must and incubated in $500-\mathrm{mL}$ bottles with orbital agitation $(120 \mathrm{rpm})$ at $28^{\circ} \mathrm{C}$. Fermentations were carried out in triplicate. Sampling was 
done at different time points of the growth phases. For harvesting, 10 OD cultures (equivalent to $10^{8}$ cells) were centrifuged at $7800 \mathrm{rpm}$ for $5 \mathrm{~min}$ in 50-mL centrifuge tubes (Sigma 2-16, Rotor 12151, Germany). Pellets were washed with Milli-Q water, frozen with liquid nitrogen and stored at $-80^{\circ} \mathrm{C}$ until melatonin analysis. Supernatants were stored at $-20^{\circ} \mathrm{C}$ until melatonin analysis was performed.

In the second experiment, different fermentation parameters were modified, using the conditions implemented with the QA23 strain in the first experiment $(200 \mathrm{~g} / \mathrm{L}, 300 \mathrm{mg} \mathrm{N} / \mathrm{L})$ and $10^{6} \mathrm{cell} / \mathrm{mL}$ and $28^{\circ} \mathrm{C}$ as control fermentations. From precultures in YPD and YNB, the QA23 strain was inoculated in $45 \mathrm{~mL}$ of standard medium in 50-mL flasks. All the conditions were tested by triplicate. To study the effect of nutrients (sugar and nitrogen) on melatonin production, the final concentration of sugars and assimilable nitrogen were decreased in some fermenters to $20 \mathrm{~g} / \mathrm{L}$ and $100 \mathrm{mg} \mathrm{N} / \mathrm{L}$, respectively. To analyze the effect of environmental parameters, inoculum size was fixed at $10^{7}$ and $10^{8} \mathrm{cell} / \mathrm{mL}$, and fermentation temperature was adjusted to 4 or $12{ }^{\circ} \mathrm{C}$. Cell cycle synchronization (arrested and not-arrested cells) was performed by adding alpha factor $(5 \mu \mathrm{g} / \mathrm{mL}$ (Sigma Aldrich, St. Louis, MO, USA)), in the haploid QA23, before inoculation in grape must.

In all fermentations, samples (10 OD) for intracellular melatonin determination were taken every $10 \mathrm{~min}$ (except for synchronization, for which time points were every $20 \mathrm{~min}$ ) and centrifuged at $7800 \mathrm{rpm}$ for $3 \mathrm{~min}$ in 50-mL centrifuge tubes (Sigma 2-16, Rotor 12151, Germany). After centrifugation, pellets were washed with Milli-Q water, frozen with liquid nitrogen and stored at $-80{ }^{\circ} \mathrm{C}$ until melatonin analysis was performed.

\subsection{Melatonin Analysis}

Melatonin samples were prepared and analyzed as described in Morcillo-Parra et al. [17]. Briefly, intracellular metabolites were extracted by adapting a boiling-buffered ethanol method [31], in which cells were incubated with $1 \mathrm{~mL}$ of a solution of $75 \%(v / v)$ boiling absolute ethanol containing $70 \mathrm{mM}$ HEPES buffer ( $\mathrm{pH} 7.5$ ) for $3 \mathrm{~min}$ at $80{ }^{\circ} \mathrm{C}$, then, concentrated by evaporation at $45^{\circ} \mathrm{C}$ in a SpeedBack (Concentrator plus, Eppendorf Ibérica, Madrid, Spain), and resuspended in $1 \mathrm{~mL}$ of Milli-Q water and centrifuged for $10 \mathrm{~min}$ at $5000 \mathrm{rpm}$. Finally, the supernatant was transferred to a new tube and stored at $-20^{\circ} \mathrm{C}$ until use.

Both extracellular and intracellular melatonin samples were extracted with a chloroform method. In brief, $50 \mu \mathrm{L}$ of sample was mixed with Milli-Q water (1:1, v:v). Then, $500 \mu \mathrm{L}$ of chloroform was added, and the mixture was shaken for $1 \mathrm{~h}$ at $1200 \mathrm{rpm}$. The organic phase was dried under a flow of nitrogen gas and resuspended in $50 \mu \mathrm{L}$ of a methanol and water mixture $(40: 60, \mathrm{v}: \mathrm{v})$. Then, samples were centrifuged for $5 \mathrm{~min}$ at 14,500 rpm and analyzed by performing liquid chromatography mass spectrometry (LC-MS/MS) as described in Morcillo-Parra et al. [17]. The system was based on high-performance liquid chromatography coupled to a triple quadrupole mass spectrometer (Agilent G6410; Agilent Technologies, Palo Alto, CA, USA), using an Agilent $150 \times 2.1 \mathrm{~mm}$ i.d., $3.5 \mu \mathrm{M}$, Zorbax Sb-Aq column in a binary gradient consisting of (A) water and (B) methanol as solvents, both containing $0.1 \%(v / v)$ formic acid. The elution profile was $100 \%$ B (4 min), $10 \%$ B (6 min). The temperature was set at $40{ }^{\circ} \mathrm{C}$, the flow rate was $0.4 \mathrm{~mL} / \mathrm{min}$ and the injection volume was $7 \mu \mathrm{L}$. The ESI conditions were as follows: a drying gas temperature of $30^{\circ} \mathrm{C}$, a flow of $12 \mathrm{~L} / \mathrm{min}$, and a nebulizer gas pressure of 25 psi. The capillarity voltage was set up at $2500 \mathrm{~V}$. Acquisition was done in positive polarity. Triple quadrupole operated in multiple reaction monitoring mode, applying a fragmentor voltage of $135 \mathrm{~V}$ and a cell accelerator voltage of $65 \mathrm{~V}$.

Melatonin quantification was performed using Agilent MassHunter WorkStation Software Quantitative Analysis Version B0104 (Agilent Technologies, Palo Alto, CA, USA) by comparing the 233/174 transition MS data of the sample and the standard.

\subsection{Statistical Analysis}

Data are expressed as the mean and standard deviation of triplicates. ANOVA and Fisher and Tukey's test analysis using XLSTAT 2020 software (Addinsoft, New York, NY, USA) were performed 
to evaluate the effect of each condition in melatonin synthesis (Tables S5-S10). The results were considered statistically significant at a $p$-value lower than 0.05. The GraphPad Prism 7 program (GraphPad software, San Diego, CA, USA) was used for graphical data modeling.

\section{Results and Discussion}

\subsection{Effect of Strain on Melatonin Synthesis}

To determine whether melatonin production during alcoholic fermentation is a general trait of Saccharomyces strains or if instead it is a strain-dependent trait or linked to the strain origin, fermentations with four strains from different environments (QA23, a wine yeast; Instaferm, a baking yeast; Levucell, an animal food yeast; Diamond, a beer yeast) were carried out.

In fact, some differences were observed in terms of growth during alcoholic fermentation (Figure 1). All yeast strains presented similar growth, except the Instaferm strain, which had lower maximal growth. However, all growth phases occurred at similar time points. In terms of fermentation performance, Diamond and QA23 consumed all sugar after three days of fermentation, as observed in the study performed by Lleixà et al. [32] under similar conditions, while Levucell and Instaferm needed one more day to consume all sugar. Both yeast strains are not used for ethanol production, which explains this slower fermentative behavior. These results were similar to those of Albertin et al. [33], who described that wine strains in enology medium are able to consume all sugars, while other food origin yeasts displayed slow or incomplete fermentations.

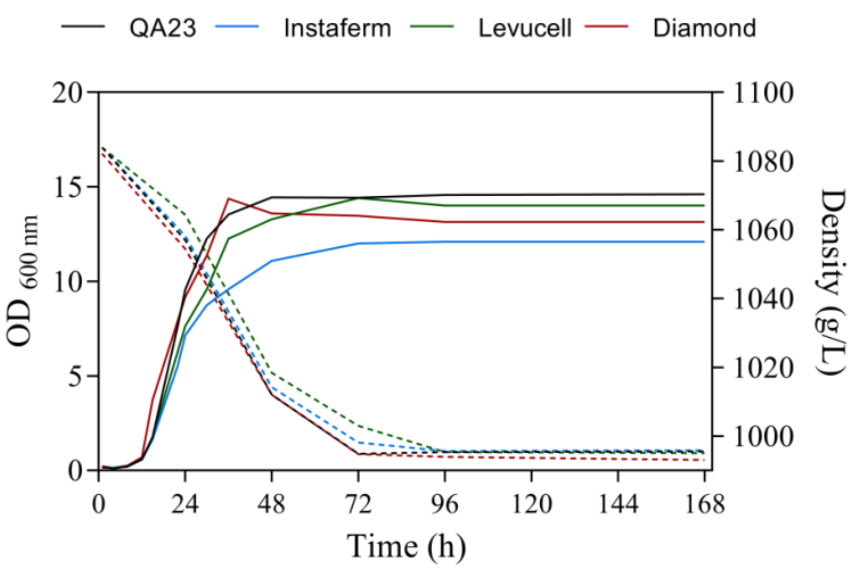

Figure 1. Fermentation kinetics of the different Saccharomyces yeast strains by monitoring the density of the must (dotted line) and yeast population, measured as optical density at $600 \mathrm{~nm}\left(\mathrm{OD}_{600}\right.$, solid line) throughout the fermentation.

In addition to the fermentation kinetics, intracellular and extracellular melatonin were measured by LC-MS/MS. On one hand, melatonin was produced intracellularly during the lag phase of yeast growth (Figure 2, Table S1), with QA23 showing the fastest synthesis $(1 \mathrm{~h})$, whereas the other Saccharomyces yeasts reached their maximum at $4 \mathrm{~h}$, as we had already observed in Saccharomyces and non-Saccharomyces yeasts in similar conditions $[17,24]$.

Even though melatonin peaks appeared at different time points ( 1 and $4 \mathrm{~h}$ ), it is important to highlight that intracellular melatonin was observed during lag phase in all yeast strains, reinforcing the relationship of melatonin with the yeast growth curve and yeast adaptation to the medium, as previously described by Rodríguez-Naranjo et al. [26]. In fact, in that study, melatonin was proposed as a signal molecule in yeast cells, linked to yeast growth phases and medium adaptation, even though it was only based on extracellular melatonin [26]. Although all yeast strains produced intracellular melatonin with a similar profile, melatonin quantities clearly differed between strains (Figure 3, Tables S1, S5 and S6), with Levucell and Diamond synthesizing more melatonin and presenting a 
basal concentration throughout all fermentations (Figure 3a). Additionally, extracellular melatonin was detected during alcoholic fermentation in all Saccharomyces strains tested (Figure 2). The QA23 and Levucell strains reached their maximum level at $24 \mathrm{~h}$, confirming previous results $[17,25,26]$. However, in the QA23 strain, the quantity of melatonin decreased from this point until $72 \mathrm{~h}$ when a synthesis rebound was observed, whereas in the Levucell strain no more melatonin was detected throughout fermentation. Conversely, Diamond exhibited its extracellular melatonin peak later, at $48 \mathrm{~h}$. However, a smaller peak was also detected at $24 \mathrm{~h}$, similar to QA23 and Levucell strains. Finally, in the Instaferm strain, melatonin content progressively increased until the end of fermentation (at 7 days), being the only one that accumulated melatonin during fermentation. These results were similar to those reported by Fernández-Cruz et al. [34], in which six Saccharomyces strains produced melatonin at different points of alcoholic fermentation.

Although the extracellular melatonin concentrations on the maximal point might reproduce the differences observed in the intracellular concentration, the huge variability observed did not allow to set statistical significance (Figure 3b, Tables S1, S7 and S8). This variability is mainly due to the fast synthesis and disappearance of this molecule, which is sometimes difficult to detect with precision.

As intracellular melatonin synthesis occurred during lag phase, and we proposed that this synthesis was related to the adaptation of yeast cells to the medium, we modified different important fermentation parameters, such as sugar and nitrogen concentrations, temperature or initial population, to determine if any of these parameters were responsible for triggering melatonin synthesis. Intracellular melatonin was monitored during the first $4 \mathrm{~h}$ of growth, covering the part of lag phase in which intracellular synthesis was detected in the previous experiment. The results were expressed as a heat map (Figure 4).
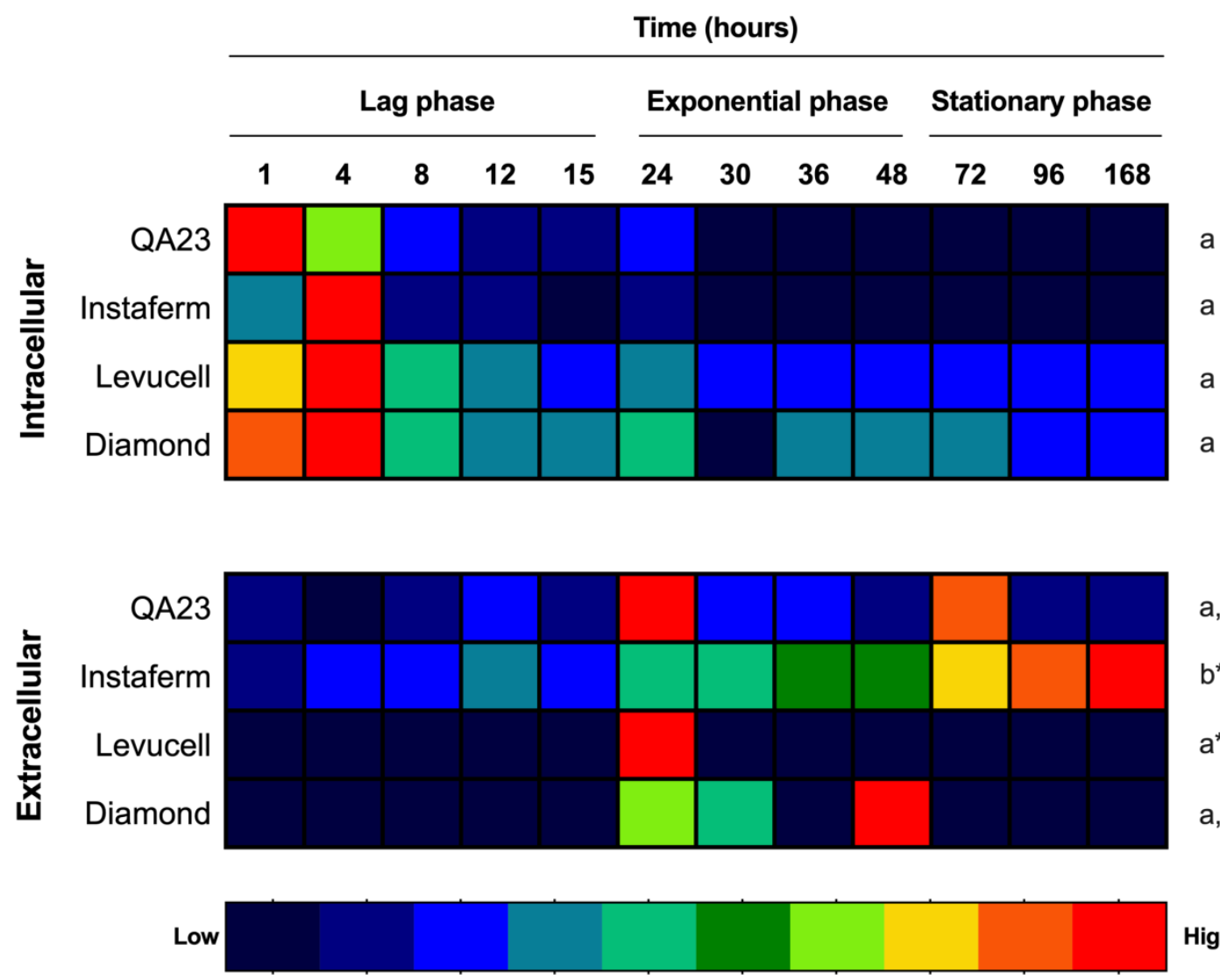

High

Figure 2. Heatmap of melatonin production by the four Saccharomyces strains during the different yeast growth phases. Concentrations were normalized to its maximum level. Different letters indicate significant differences between conditions within each strain, $p<0.05$. 
a)

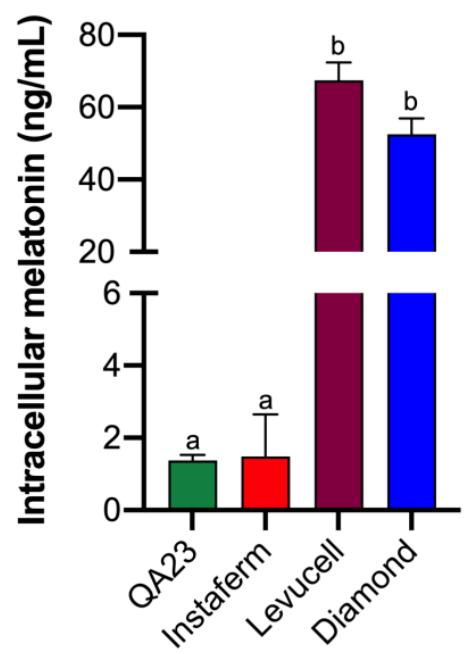

Strains b)

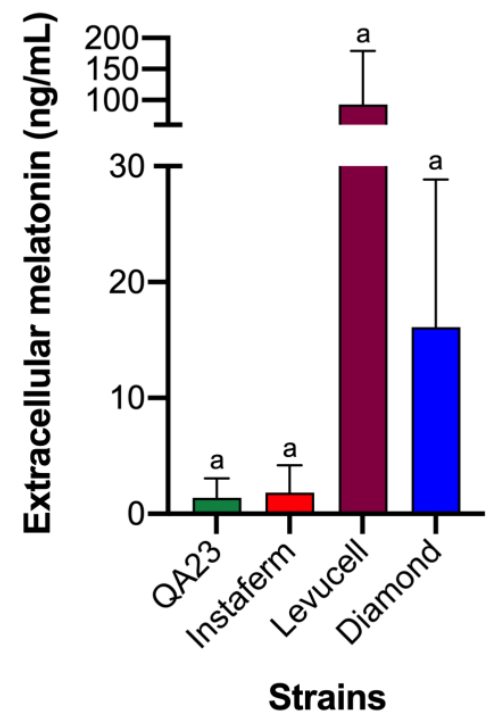

Figure 3. Maximal intracellular (a) and extracellular (b) melatonin concentration by different Saccharomyces strains. Intracellular melatonin is expressed in $\mathrm{ng} / \mathrm{mL}$ per $10 \mathrm{OD}$. Error bars represent \pm SD of $n=3$ by ANOVA. Different letters indicate significant differences between strains. $p<0.05$.

Time (minutes)

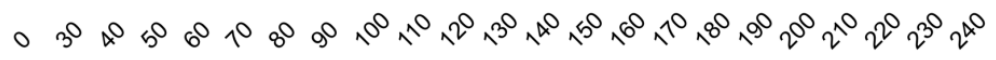

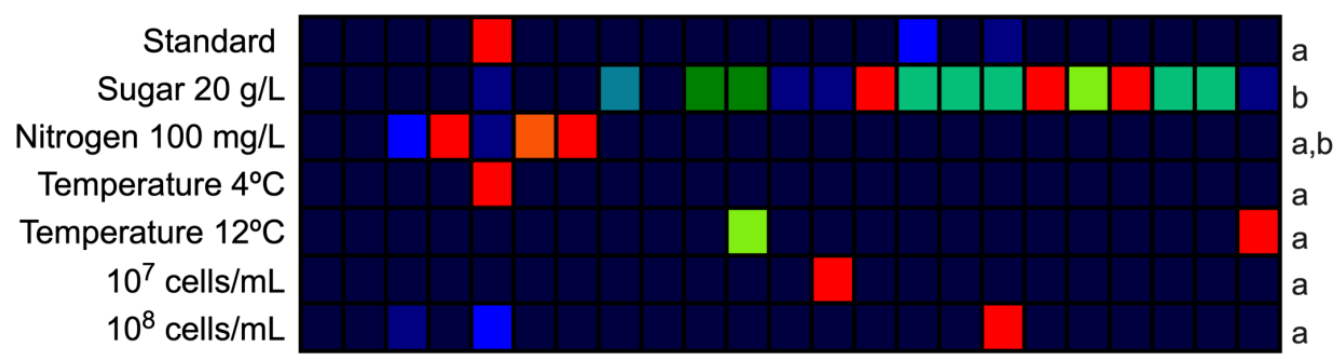

Low

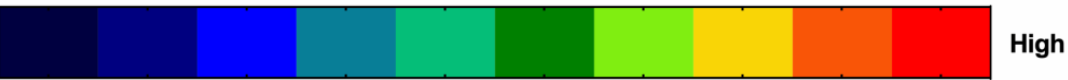

Figure 4. Heatmap of melatonin production in several fermentation conditions in S. cerevisiae QA23: Low sugar (20 g/L), low nitrogen (100 mg N/L) concentration, different temperatures and inoculum amount. Concentrations were normalized to its maximum level. Standard fermentation was grape must with $200 \mathrm{~g} / \mathrm{L}$ sugar and $300 \mathrm{mg} \mathrm{N} / \mathrm{L}$, inoculated at $10^{6}$ cells $/ \mathrm{mL}$ and fermented at $28^{\circ} \mathrm{C}$. Different letters indicate significant differences between conditions, $p<0.05$.

\subsection{Effects of Sugar and Nitrogen on Melatonin Synthesis}

As explained in the previous experiment, QA23 in standard conditions $(200 \mathrm{~g} / \mathrm{L}$ sugar and $300 \mathrm{mg} \mathrm{N} / \mathrm{L}$ ) showed a peak on intracellular melatonin at $60 \mathrm{~min}$ (Figure 4). However, in low-sugar conditions $(20 \mathrm{~g} / \mathrm{L})$, intracellular melatonin was detected starting $90 \mathrm{~min}$ after inoculation and increasing progressively until 150, 190 and $210 \mathrm{~min}$, when the maximum melatonin concentration was quantified. In contrast, in low-nitrogen conditions, intracellular melatonin had a similar profile as that in standard conditions, appearing in a similar timeframe (50 and $80 \mathrm{~min}$ ), although it remained 
longer in the intracellular medium. Even though we observed differences in the time of detection, in which intracellular melatonin is synthetized, we did not observe those differences in the amount of melatonin produced, again due to the high dispersion between triplicates in some samples (Figure 5, Tables S2, S9 and S10). Thus, only glucose limitation affected melatonin synthesis profile during alcoholic fermentation. This result may highlight osmotic stress as being responsible for intracellular melatonin synthesis, but also a change in the carbon metabolism of yeast due to the low sugar concentration. In fact, recent results of our group have shown that melatonin interacts with some glycolytic proteins in yeast with high fermentative capacity, pinpointing a possible role of melatonin as a signal molecule, likely related to fermentation metabolism [17,24]. Therefore, the quantity of sugar present in the medium, which can determine the metabolism of sugars in yeast, could be a key parameter in melatonin synthesis or function.

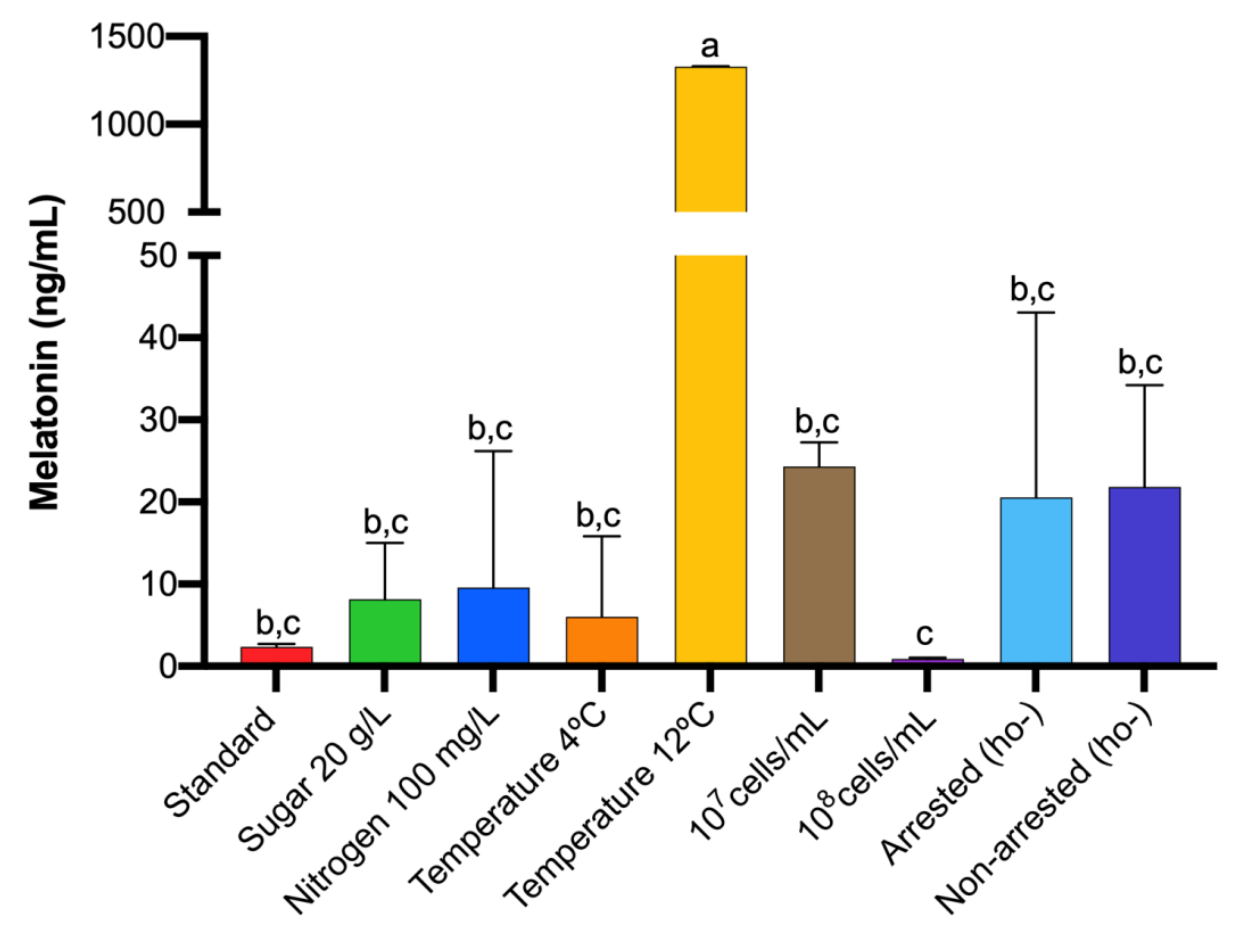

Figure 5. Maximal intracellular melatonin concentration by S. cerevisiae QA23 in several fermentation conditions: Low sugar ( $20 \mathrm{~g} / \mathrm{L})$, low nitrogen ( $100 \mathrm{mg} \mathrm{N} / \mathrm{L})$ concentration, temperature, inoculum amount, arrested and non-arrested cells. Data are expressed in $\mathrm{ng} / \mathrm{mL}$ per $10^{8}$ cells. Standard fermentation was grape must with $200 \mathrm{~g} / \mathrm{L}$ sugar and $300 \mathrm{mg} \mathrm{N} / \mathrm{L}$, inoculated at $10^{6}$ cells $/ \mathrm{mL}$ and fermented at $28^{\circ} \mathrm{C}$. Error bars represent $\pm \mathrm{SD}$ of $n=3$ by ANOVA. Different letters indicate significant differences between conditions, $p<0.05$.

\subsection{Effect of Temperature on Melatonin Synthesis}

To elucidate the effect of temperature in melatonin production, alcoholic fermentations were performed at 4 and $12{ }^{\circ} \mathrm{C}$, two extreme temperatures, in which a longer lag phase and therefore, a delay in the alcoholic fermentation is expected, in comparison to standard fermentation carried out at $28{ }^{\circ} \mathrm{C}$.

Intracellular melatonin was detected at all temperatures tested (Figure 4, Table S3). However, surprisingly, higher differences were observed in melatonin synthesis at 12 than $4{ }^{\circ} \mathrm{C}$ (Figure 5, Tables S9 and S10). On melatonin production profile, it peaked at $240 \mathrm{~min}$ at $12^{\circ} \mathrm{C}$, whereas at the other two temperatures, the highest synthesis was observed at $60 \mathrm{~min}$. Additionally, another peak of lower concentration appeared at $120 \mathrm{~min}$ at both low temperatures $\left(4\right.$ and $\left.12{ }^{\circ} \mathrm{C}\right)$. Temperature is an important factor during alcoholic fermentation that affects its development, as well as yeast growth, being slower at lower temperatures $[9,10]$. The results at $12{ }^{\circ} \mathrm{C}$ seemed to point towards a delayed effect 
on melatonin synthesis due to the prolonged lag phase at low temperatures. In fact, Wang et al. [28] observed that fermentations carried out at $16{ }^{\circ} \mathrm{C}$ delayed and decreased melatonin production in mulberry wines. However, the results at $4{ }^{\circ} \mathrm{C}$ did not support this delay effect; it may be that extreme temperatures such as $4{ }^{\circ} \mathrm{C}$ triggered other mechanisms responsible for this early melatonin synthesis.

\subsection{Effect of the Initial Yeast Population on Melatonin Synthesis}

To understand the effect of inoculum amount in melatonin production, fermentations with $10^{7}$ and $10^{8}$ cells $/ \mathrm{mL}$ were carried out in comparison with the standard inoculation rate $\left(10^{6} \mathrm{cell} / \mathrm{mL}\right)$. As mentioned above, in standard inoculation conditions, melatonin was detected at 60 min (Figure 4). When the inoculum was increased, melatonin synthesis was delayed at $140 \mathrm{~min}$ for $10^{7} \mathrm{cell} / \mathrm{mL}$ and $180 \mathrm{~min}$ for $10^{8} \mathrm{cell} / \mathrm{mL}$. Therefore, the higher the population we used, the greater the delay of the intracellular melatonin peak we obtained. Additionally, melatonin content was higher in $10^{7}$ cell $/ \mathrm{mL}$ inoculum (Figure 5, Tables S3, S9 and S10). Given that high inoculum size causes less growth of cells [35], this delay in melatonin synthesis may add more evidences that point out melatonin as a signal molecule for yeast growth.

\subsection{Effect of Cell Cycle Synchronization on Melatonin Synthesis}

Finally, as we obtained high variability in melatonin concentration between triplicates, we wanted to synchronize the cell cycle to have most cells in the same cell cycle phase and thus decrease this variability. Due to the impossibility of synchronizing QA23, the correspondent haploid was studied (QA23 ho-). Treatment with alpha-factor was used to synchronize the cell cycle in G1 phase.

However, despite synchronizing $85 \%$ of cells, we did not achieve a clear decrease in melatonin detection variability (Figure 5, Tables S4, S9 and S10), probably due to its fast appearance and disappearance and the impossibility of monitoring its synthesis in smaller periods.

\section{Conclusions}

Although differences were observed between strains, in this study, we can confirm that the pattern of melatonin synthesis described for the strain S. cerevisiae QA23 in a previous study [17] is also observed in the rest of strains of Saccharomyces. Therefore, the production of intracellular melatonin during the lag phase of yeast growth and its secretion to the medium in the mid-end exponential or early stationary phases seem to be a species-specific pattern and not strain dependent. Moreover, differences between strains can be attributed to their different isolation origin or uses, and therefore, to different adaptation mechanisms to the fermentation medium, as Vigentini et al. [14] described for Torulaspora delbrueckii and S. cerevisiae strains.

When several conditions that affect the performance of alcoholic fermentation were tested, the intracellular melatonin peak was delayed in a low sugar concentration, a fermentation temperature of $12{ }^{\circ} \mathrm{C}$ and with a higher initial population. Nevertheless, in all conditions, melatonin appeared during lag phase and as a rapid signal molecule. It is also important to highlight that melatonin quantities were very different between conditions, occurring the highest production when the fermentation temperature was fixed at $12^{\circ} \mathrm{C}$.

Finally, despite the fact that we were not able to clearly unravel which conditions trigger melatonin synthesis in yeast cells during alcoholic fermentation, we observed that sugar content has the most apparent effect on melatonin synthesis. These results together with previous ones of the group, in which interactions of melatonin with glycolytic proteins were described $[17,24]$, seem to clearly indicate an active role of this molecule in the sugar metabolism, specifically in fermentative metabolism. Nevertheless, further studies are needed to confirm this hypothesis and to understand the exact mechanism and the triggering of melatonin synthesis.

Supplementary Materials: The following are available online at http://www.mdpi.com/2076-2607/8/6/853/s1, Table S1: Intracellular and extracellular melatonin produced by the four different Saccharomyces strains during alcoholic fermentation, Table S2: Intracellular melatonin production by S. cerevisiae QA23 in a standard, low-glucose 
and -nitrogen grape must, Table S3: Effect of low temperature and inoculum size on intracellular melatonin production by S. cerevisiae QA23, Table S4: Comparison of intracellular melatonin production by arrested and non-arrested yeast cells, Table S5: Analysis of differences between intracellular melatonin synthesis by different Saccharomyces strains, Table S6: Analysis of differences between intracellular melatonin synthesis profile by different Saccharomyces strains, Table S7: Analysis of differences between extracellular melatonin synthesis by different Saccharomyces strains, Table S8: Analysis of differences between extracellular melatonin synthesis profile by different Saccharomyces strains, Table S9: Analysis of differences between intracellular melatonin synthesis on different conditions, Table S10: Analysis of differences between intracellular melatonin synthesis profile on different conditions.

Author Contributions: Conceptualization, A.M., G.B. and M.-J.T.; methodology M.Á.M.-P.; investigation, M.Á.M.-P.; writing—original draft preparation, M.Á.M.-P.; writing—review and editing, A.M., G.B. and M.-J.T.; supervision, A.M., G.B. and M.-J.T.; resources, A.M., G.B. and M.-J.T.; funding acquisition, A.M., G.B. and M.-J.T. All authors have read and agreed to the published version of the manuscript.

Funding: The work was supported by the Ministry of Economy and Competitiveness, Spain (Projects AGL2013-47300-C3-1-R and AGL2016-77505-C3-3-R), for financial support. M.A.M.-P. holds a fellowship linked to AGL2013-47300-C3-1-R Project.

Acknowledgments: Thanks to Rosa Ras from Centre for Omic Sciences (COS) Join Unit of the Universitat Rovira i Virigili-Eurecat and Irene Maijó and Sònia Abelló from Servei de Recursos Científics i Tècnics of Universitat Rovira i Virgili for technical assistance in LC-MS/MS analysis.

Conflicts of Interest: The authors declare no conflict of interest.

\section{References}

1. Zara, G.; van Vuuren, H.J.J.; Mannazzu, I.; Zara, S.; Budroni, M. Transcriptomic response of Saccharomyces cerevisiae during fermentation under oleic acid and ergoesterol depletion. Fermentation 2019, 5, 57. [CrossRef]

2. Sommer, S. Monitoring the functionality and stress response of yeast cells using flow citometry. Microorganisms 2020, 8, 619. [CrossRef] [PubMed]

3. Bordet, F.; Joran, A.; Klein, G.; Roullier-Gall, C.; Alexandre, H. Yeast-yeast interactions: Mechanisms, methodologies and impact on composition. Microorganisms 2020, 8, 600. [CrossRef] [PubMed]

4. Peña, R.; Chávez, R.; Rodríguez, A.; Ganga, M.A. A control alternative for the hidden enemy in the wine cellar. Fermentation 2019, 5, 25. [CrossRef]

5. Heinisch, J.J.; Rodicio, R. Stress responses in wine yeast. In Biology of Microorganisms on Grapes, in Must and in Wine; Köning, H., Unden, G., Fröhlich, J., Eds.; Springer: Cham, Switzerland, 2017; pp. 377-395.

6. Bauer, E.; Pretorius, L. Yeast stress response and fermentation efficiency: How to survive the making of wine-A Review. S. Afr. J. Enol. Vitic. 2000, 21, 27-51. [CrossRef]

7. Hohmann, S. Yeast stress responses. In Yeast Stress Response; Hohmann, S., Mager, W.H., Eds.; R.G. Landes: Austin, TX, USA, 1997; pp. 101-145.

8. Bisson, L.F. Stuck and sluggish fermentations. Am. J. Enol. Vitic. 1999, 50, 107-119.

9. Beltran, G.; Novo, M.; Guillamón, J.M.; Mas, A.; Rozès, N. Effect of fermentation temperature and culture media on the yeast lipid composition and wine volatile compounds. Int. J. Food Microbiol. 2008, 121, $169-177$. [CrossRef] [PubMed]

10. Torija, M.J.; Beltran, G.; Novo, M.; Poblet, M.; Guillamón, J.M.; Mas, A.; Rozes, N. Effects of fermentation temperature and Saccharomyces species on the cell fatty acid composition and presence of volatile compounds in wine. Int. J. Food Microbiol. 2003, 85, 127-136. [CrossRef]

11. Vázquez, J.; González, B.; Sempere, V.; Mas, A.; Torija, M.J.; Beltran, G. Melatonin reduces oxidative stress damage induced by hydrogen peroxide in Saccharomyces cerevisiae. Front. Microbiol. 2017, 8, 1066. [CrossRef]

12. Vázquez, J.; Grillitsch, K.; Daum, G.; Mas, A.; Torija, M.J.; Beltran, G. Melatonin minimizes the impact of oxidative stress induced by hydrogen peroxide in Saccharomyces and non-conventional yeast. Front. Microbiol. 2018, 9, 1933. [CrossRef]

13. Bisquert, R.; Muñiz-Calvo, S.; Guillamón, J.M. Protective role of intracellular melatonin against oxidative stress and UV radiation in Saccharomyces cerevisiae. Front. Microbiol. 2018, 9, 318. [CrossRef] [PubMed]

14. Vigentini, I.; Gardana, C.; Fracassetti, D.; Gabrielli, M.; Foschino, R.; Simonetti, P.; Tirelli, A.; Iriti, M. Yeast contribution to melatonin, melatonin isomers and tryptophan ethyl ester during alcoholic fermentation of grape musts. J. Pineal Res. 2015, 58, 388-396. [CrossRef] [PubMed] 
15. Fernández-Cruz, E.; Cerezo, A.B.; Cantos-Villar, E.; Troncoso, A.M.; García-Parrilla, M.C. Time course of L-tryptophan metabolites when fermenting natural grape musts: Effect of inoculation treatments and cultivar on the occurrence of melatonin and related indolic compounds. Aust. J. Grape Wine Res. 2019, 25, 92-100. [CrossRef]

16. Fernandez-Cruz, E.; González, B.; Muñiz-Calvo, S.; Morcillo-Parra, M.Á.; Bisquert, R.; Troncoso, A.M.; Garcia-Parrilla, M.C.; Torija, M.J.; Guillamón, J.M. Intracellular biosynthesis of melatonin and other indolic compounds in Saccharomyces and non-Saccharomyces wine yeasts. Eur. Food Res. Technol. 2019, 245, 1553-1560. [CrossRef]

17. Morcillo-Parra, M.A.; Valera, M.J.; Beltran, G.; Mas, A.; Torija, M.J. Glycolytic proteins interact with intracellular melatonin in Saccharomyces cerevisiae. Front. Microbiol. 2019, 10, 2424. [CrossRef]

18. Sprenger, J.; Hardeland, R.; Fuhrberg, B.; Han, S.Z.M. Melatonin and other 5-methoxylated indoles in yeast: Presence in high concentrations and dependence on tryptophan availability. Cytologia 1999, 64, 209-213. [CrossRef]

19. Garcia-Moreno, H.; Calvo, J.R.; Maldonado, M.D. High levels of melatonin generated during the brewing process. J. Pineal Res. 2013, 55, 26-30. [CrossRef] [PubMed]

20. Kocadağlı, T.; Yılmaz, C.; Gökmen, V. Determination of melatonin and its isomer in foods by liquid chromatography tandem mass spectrometry. Food Chem. 2014, 153, 151-156. [CrossRef]

21. Rodriguez-Naranjo, M.I.; Gil-Izquierdo, A.; Troncoso, A.M.; Cantos, E.; García-Parrilla, M.C. Melatonin: A new bioactive compound in wine. J. Food Compos. Anal. 2011, 24, 603-608. [CrossRef]

22. Rodríguez-Naranjo, M.I.; Gil-Izquierdo, A.; Troncoso, A.M.; Cantos-Villar, E.; Garcia-Parrilla, M.C. Melatonin is synthesised by yeast during alcoholic fermentation in wines. Food Chem. 2011, 126, 1608-1613. [CrossRef]

23. Fernández-Cruz, E.; Álvarez-Fernández, M.A.; Valero, E.; Troncoso, A.M.; García-Parrilla, M.C. Melatonin and derived L-tryptophan metabolites produced during alcoholic fermentation by different wine yeast strains. Food Chem. 2017, 217, 431-437. [CrossRef]

24. Morcillo-Parra, M.A.; González, B.; Beltran, G.; Mas, A.; Torija, M.J. Melatonin and glycolytic protein interactions are related to yeast fermentative capacity. Food Microbiol. 2020, 87, 103398. [CrossRef] [PubMed]

25. Valera, M.J.; Morcillo-Parra, M.A.; Zagórska, I.; Mas, A.; Beltran, G.; Torija, M.J. Effects of melatonin and tryptophol addition on fermentations carried out by Saccharomyces cerevisiae and non-Saccharomyces yeast species under different nitrogen conditions. Int. J. Food Microbiol. 2019, 89, 174-181. [CrossRef] [PubMed]

26. Rodríguez-Naranjo, M.I.; Torija, M.J.; Mas, A.; Cantos-Villar, E.; Garcia-Parrilla, M.C. Production of melatonin by Saccharomyces strains under growth and fermentation conditions. J. Pineal Res. 2012, 53, $219-224$. [CrossRef]

27. Muñiz-Calvo, S.; Bisquert, R.; Fernández-Cruz, E.; García-Parrilla, M.C.; Guillamón, J.M. Deciphering the melatonin metabolism in Saccharomyces cerevisiae by the bioconversion of related metabolites. J. Pineal Res. 2019, 66, e12554. [CrossRef]

28. Wang, C.; Yin, L.Y.; Shi, X.Y.; Xiao, H.; Kang, K.; Liu, X.Y.; Zhan, J.C.; Huang, W.D. Effect of cultivar, temperature, and environmental conditions on the dynamic change of melatonin in mulberry fruit development and wine fermentation. J. Food Sci. 2016, 81, 958-967. [CrossRef] [PubMed]

29. Chiva, R.; López-Malo, M.; Salvó, Z.; Mas, A.; Guillamón, J.M. Analysis of low temperature-induced genes (LTIG) in wine yeast during alcoholic fermentation. FEMS Yeast Res. 2012, 12, 831-843. [CrossRef]

30. Beltran, G.; Novo, M.; Rozès, N.; Mas, A.; Guillamón, J.M. Nitrogen catabolite repression in Saccharomyces cerevisiae during wine fermentations. FEMS Yeast Res. 2004, 4, 625-632. [CrossRef]

31. Gonzalez, B.; François, J.; Renaud, M. A rapid and reliable method for metabolite extraction in yeast using boiling buffered ethanol. Yeast. 1997, 13, 1347-1356. [CrossRef]

32. Lleixà, J.; Martín, V.; Giorello, F.; Portillo, M.C.; Carrau, F.; Beltran, G.; Mas, A. Analysis of the NCR Mechanisms in Hanseniaspora vineae and Saccharomyces cerevisiae during winemaking. Front. Genet. 2019, 9, 747. [CrossRef]

33. Albertin, W.; Marullo, P.; Aigle, M.; Dillmann, C.; de Vienne, D.; Bely, M.; Sicard, D. Population size drives industrial Saccharomyces cerevisiae alcoholic fermentation and is under genetic control. Appl. Environ. Microbiol. 2011, 77, 2772-2784. [CrossRef] [PubMed] 
34. Fernández-Cruz, E.; Álvarez-Fernández, M.A.; Valero, E.; Troncoso, A.M.; García-Parrilla, M.C. Validation of an analytical method to determine melatonin and compounds related to L-tryptophan metabolism using UHPLC/HRMS. Food Anal. Method. 2016, 9, 3327-3336. [CrossRef]

35. Carrau, F.; Medina, K.; Fariña, L.; Boido, E.; Dellacassa, E. Effect of Saccharomyces cerevisiae inoculum size on wine fermentation aroma compounds and its relation with assimilable nitrogen content. Int. J. Food Microbiol. 2010, 43, 81-85. [CrossRef] [PubMed]

C 2020 by the authors. Licensee MDPI, Basel, Switzerland. This article is an open access article distributed under the terms and conditions of the Creative Commons Attribution (CC BY) license (http://creativecommons.org/licenses/by/4.0/). 